\title{
Gradients socioéconomiques du risque cardiovasculaire chez les enfants et les adolescents canadiens
}

\author{
Y. Shi, M.D., Ph. D.; M. de Groh, Ph. D.; C. Bancej, Ph. D.
}

Cet article a fait l'objet d'une évaluation par les pairs.

Diffuser cet article sur Twitter

\section{Résumé}

Introduction : Les maladies cardiovasculaires (MCV) et leurs facteurs de risque présentent des gradients socioéconomiques clairs chez les adultes canadiens, mais présentent des ambiguïtés chez les enfants. L'objectif de cette étude est de vérifier l'existence ou non de gradients socioéconomiques dans les marqueurs physiologiques du risque de MCV chez les enfants et les adolescents canadiens.

Méthodologie : À partir des données transversales combinées de l'Enquête canadienne sur les mesures de la santé 2007-2011, nous avons étudié, chez 2149 enfants (6 à 11 ans) et 2073 adolescents (12 à 17 ans) et selon le sexe, les marqueurs de risque cardiovasculaire suivants : excès de poids (y compris l'obésité), score de capacité aérobique (SCA), pression artérielle (PA), lipides sanguins (totaux, cholestérol LDL et HDL, triglycérides), métabolisme du glucose et protéine $\mathrm{C}$ réactive (CRP). Des analyses de régression logistique et de régression linéaire multidimensionnelles ont permis de dégager les tendances relatives au risque cardiovasculaire en fonction de la suffisance du revenu du ménage et du niveau de scolarité des parents, après ajustement en fonction de l'âge et de l'origine ethnique, et après stratification par groupe d'âge et par sexe.
Principales constatations

- La prévalence de l'obésité s'est révélée plus élevée chez les garçons que chez les filles.

- Les garçons étaient en meilleure condition physique que les filles.

- Les enfants et les adolescents canadiens, et plus particulièrement les filles, présentent des gradients socioéconomiques significatifs en ce qui concerne l'obésité, la condition physique et plusieurs marqueurs physiologiques du risque de maladie cardiovasculaire.

Résultats : La prévalence de l'obésité était sensiblement plus élevée chez les jeunes garçons que chez les jeunes filles (prévalence de 18,5 \%, intervalle de confiance [IC] à $95 \%$ : 15,6 à 21,5 contre 7,7\%, IC à $95 \%: 5,2$ à 10,3). Toutefois, des gradients socioéconomiques négatifs ont été observés en ce qui concerne le risque d'adiposité chez les jeunes filles et les adolescentes, et non chez les garçons. Parmi les enfants et les adolescents, les garçons étaient en meilleure condition physique que les filles (SCA moyen de 541, IC à $95 \%: 534$ à 546 contre 501 , IC à $95 \%: 498$ à 505 chez les enfants; 522, IC à $95 \%$ : 514 à 529 contre 460, IC à $95 \%$ : 454 à 466 chez les adolescents; $p<0,001$ ). Bien qu'un gradient positif lié au revenu ait été observé relativement au SCA tant chez les garçons que chez les filles, la signification statistique a été atteinte seulement chez les filles ( $p=0,006)$. Un gradient négatif a été observé en fonction du niveau de scolarité des parents relativement à la PA des jeunes enfants. Bien que nous ayons constaté des différences importantes selon le sexe en ce qui concerne la PA systolique, le cholestérol total, le cholestérol HDL, la glycémie à jeun et la CRP chez les adolescents, des gradients socioéconomiques spécifiques au sexe ont uniquement été observés pour la PA systolique, le cholestérol HDL et le cholestérol LDL. Des études fondées sur de grands échantillons devront être réalisées afin de confirmer ces observations.

Conclusion : Cette étude a révélé d'importantes différences selon le sexe et des gradients socioéconomiques en ce qui concerne l'adiposité, la capacité aérobique et les marqueurs physiologiques du risque de MCV chez les enfants canadiens d'âge scolaire. Des interventions de santé visant à atténuer les gradients socioéconomiques du risque de MCV devraient être mises en place dès l'enfance dans la population, en particulier en ce qui touche la prévention de l'obésité chez les jeunes garçons de tout statut socioéconomique (SSE) et chez les filles dont le SSE est faible, la promotion d'une bonne condition physique, en particulier auprès des filles et des jeunes de tous âges dans les groupes à faible SSE, et enfin la sensibilisation des parents, spécialement de ceux qui ont un faible niveau de scolarité, à l'égard du risque de MCV précoce chez les enfants.

Mots-clés : gradients socioéconomiques, statut socioéconomique, risque cardiovasculaire, condition physique, obésité, enfants et adolescents, Enquête canadienne sur les mesures de la santé

Rattachement des auteurs :

Direction des déterminants sociaux et de l'intégration scientifique, Agence de la santé publique du Canada, Ottawa (Ontario), Canada

Correspondance : Yipu Shi, Direction des déterminants sociaux et de l'intégration scientifique, Agence de la santé publique du Canada, Ottawa (Ontario) K1A 0K9; tél. : 613-941-2436; téléc. : 613-960-0921; courriel : yipu.shi@phac-aspc.gc.ca 


\section{Introduction}

Les maladies cardiovasculaires (MCV) constituent la première cause de décès chez les adultes canadiens ${ }^{1}$. Chez les adultes, les MCV et leurs facteurs de risque suivent des gradients socioéconomiques clairs $^{2,3}$. Ces facteurs de risque physiologiques et comportementaux associés aux MCV sont un excès de poids (incluant l'obésité), une pression artérielle (PA) élevée, un taux élevé de lipoprotéine de basse densité (LDL) et un faible taux de lipoprotéine de haute densité (HDL), une hyperglycémie, l'usage du tabac, l'inactivité physique ou une mauvaise condition physique et enfin une alimentation riche en lipides ${ }^{4}$. Les facteurs comportementaux sont associés à un grand nombre de résultats défavorables sur le plan de la santé, dont l'apparition de facteurs de risque physiologiques de $\mathrm{MCV}^{5}$. On utilise le statut socioéconomique (SSE) comme indicateur de l'environnements physique dans lequel les enfants vivent et jouent. Des données probantes récentes donnent à penser que plusieurs comportements de santé néfastes associés au risque de MCV, tels qu'une alimentation malsaine, l'inactivité physique et l'usage du tabac, sont beaucoup plus répandus chez les jeunes à faible $S E^{6-8}$. D'après les résultats d'autres travaux de recherche, le conditionnement biologique associé aux épreuves vécues pendant les périodes cruciales du développement de l'enfant peut influencer la santé de ce dernier à long terme, et les enfants à faible SSE sont susceptibles d'être particulièrement vulnérables aux circonstances stressantes ${ }^{9}$. Davantage de données probantes sont nécessaires pour vérifier l'existence de gradients socioéconomiques de la santé physique chez les enfants exposés à différents contextes socioéconomiques.

Un grand nombre de données probantes démontrent que les facteurs de risque de MCV apparaissent au cours de l'enfance et qu'un faible SSE à cette période est associé à un risque élevé de $\mathrm{MCV}$ et à une mortalité accrue par MCV plus tard dans la vie $^{10,11}$. On mesure souvent le SSE en utilisant le revenu familial, le niveau de scolarité des parents et leur situation visà-vis de l'emploi. Le revenu familial et le niveau de scolarité des parents, qui constituent deux dimensions distinctes du
SSE, comptent parmi les facteurs sociaux le plus fortement associés à la santé ${ }^{12}$. Le revenu est susceptible d'influer le plus directement sur la santé par l'accès aux ressources matérielles, le niveau de scolarité quant à lui étant directement lié à la santé par les comportements de santé et les choix relatifs au mode de vie et indirectement par le revenu et les facteurs psychosociaux ${ }^{13}$. La contribution directe et indirecte de ces facteurs matériels est un puissant élément prédictif de la santé des enfants. Des études intergénérationnelles ont révélé que le niveau de scolarité des parents est susceptible d'influencer grandement les résultats des enfants sur le plan de la santé et des études ${ }^{14}$. L'évaluation des effets respectifs du revenu et du niveau de scolarité sur la santé peut nous aider à comprendre les mécanismes par lesquels ils influent sur la santé et à proposer davantage d'options pour l'élaboration de politiques capables de réduire le risque que les enfants aient de piètres résultats sur le plan de la santé.

Alors que beaucoup de pays connaissent des épidémies d'obésité chez les enfants, différentes études ont démontré l'existence de gradients socioéconomiques concernant l'obésité, ce qui a conduit à l'élaboration et à la mise en œuvre d'interventions visant à réduire les inégalités associées à l'obésité des enfants ${ }^{15,16}$. Bien que les données récentes laissent penser qu'il existe des gradients socioéconomiques de l'excès de poids différents selon le sexe chez les adultes canadiens, les études chez les enfants n'ont mis en évidence aucune différence de ce type dans le risque d'excès de poids lié au $\mathrm{SSE}^{8,17}$. Peu d'études ont été menées sur l'émergence précoce de gradients socioéconomiques des marqueurs physiologiques du risque de $\mathrm{MCV}$, et les résultats de ces études divergent, entre autres à cause de différences dans les indicateurs du SSE et dans l'âge des populations étudiées ${ }^{18-20}$. La compréhension des facteurs socioéconomiques qui influent sur le risque de MCV pendant l'enfance pourrait nous aider à déterminer les groupes à risque élevé et à les cibler grâce à des programmes de prévention précoce des MCV en vue de prévenir les inégalités durables en ce qui concerne les MCV.

L'Enquête canadienne sur les mesures de la santé (ECMS) ${ }^{21}$, une enquête représentative à l'échelle nationale dans le cadre de laquelle s'effectuent des mesures physiques et des prélèvements d'échantillons de sang et d'urine, offre une occasion unique d'étudier l'association entre le SSE et les marqueurs physiologiques du risque de MCV chez les enfants et les adolescents canadiens.

\section{Méthodologie}

\section{Source de données}

L'ECMS est une enquête transversale exhaustive sur les mesures de la santé qui vise à recueillir de l'information sur l'état de santé et les facteurs de risque au sein des ménages canadiens. L’enquête est représentative de $96,3 \%$ de la population canadienne de 6 à 79 ans vivant à domicile dans les 10 provinces et les 3 territoires. Sont exclus de l'enquête les personnes vivant dans les réserves et les autres établissements autochtones, dans certaines régions éloignées ou en établissement, de même que les membres à temps plein des Forces armées canadiennes. Le comité d'éthique de la recherche de Santé Canada a examiné et approuvé la totalité des processus et des protocoles de l'ECMS. La participation à l'enquête était volontaire, et chaque participant a donné son consentement éclairé. Nous avons combiné les données des cycles 1 (2007-2009) et 2 (2000-2011) de l'ECMS pour la réalisation de la présente étude, avec un taux de réponse global des deux cycles combinés de $53,5 \%{ }^{21}$.

Les enquêtes ont été réalisées au moyen d'entrevues auprès de ménages qui ont répondu à des questions sur leurs caractéristiques sociodémographiques, leur état de santé et leur mode de vie et se sont rendus dans un centre d'examen mobile où des mesures physiques ont été prises et des échantillons de sang et d'urine ont été prélevés. Les parents ou les tuteurs ont répondu aux questions pour les participants de 6 à 13 ans, les participants de 14 ans et plus ayant répondu eux-mêmes. Environ la moitié des répondants ont été choisis au hasard en vue du prélèvement de sang à jeun. Afin d'accroître la puissance statistique, nous avons combiné les cycles 1 et 2 de l'ECMS, ce qui nous a permis d'obtenir un échantillon de 3799 répondants de 6 à 17 ans (dont 1693 personnes à jeun). Les données ont été recueillies entre 2007 et 2011. 


\section{Mesures}

\section{Statut socioéconomique}

Nous avons utilisé la suffisance du revenu du ménage et le niveau de scolarité des parents comme indicateurs du SSE. La suffisance du revenu du ménage est une variable calculée par Statistique Canada qui tient compte du revenu familial total, toutes sources confondues, et du nombre total de membres du ménage. Les catégories de suffisance du revenu sont les suivantes : " inférieure », " moyenne-inférieure », « moyenne-supérieure » et « supérieure ». Comme environ $70 \%$ seulement des répondants à l'ECMS ont indiqué le revenu total de leur ménage, Statistique Canada a utilisé des techniques de modélisation par régression afin d'attribuer les valeurs manquantes, en se fondant sur les données suivantes ou sur certaines d'entre elles : réponses partielles concernant la tranche de revenu, plus proche voisin, lieu de collecte et taille du ménage ${ }^{22}$. Nous avons choisi d'inclure uniquement les répondants dont l'estimation du revenu était fondée sur la tranche de revenu, qu'elle soit déclarée entièrement ou partiellement.

Le niveau de scolarité des parents correspond au plus haut niveau d'études atteint par l'un ou l'autre des parents et a été classé en fonction des catégories suivantes : " études secondaires non terminées ", « diplôme d'études secondaires », « études post-secondaires partielles » et «diplôme d'études post-secondaires ». L'origine ethnique a été catégorisée en «blanche » ou « non blanche ", cette dernière incluant les Autochtones vivant à l'extérieur des réserves. Les répondants pour lesquels il manquait des valeurs pour une ou plusieurs de ces variables ont été exclus de l'analyse. L'échantillon final était de 3591 personnes, dont 1645 répondants à jeun.

\section{Résultats sur le plan de la santé} cardiovasculaire

Le poids à la naissance des participants à l'ECMS de 6 à 11 ans a été fourni par leurs parents ou tuteurs. La pratique de l'activité physique des enfants a été déterminée à partir de la réponse du parent ou du tuteur à la question « Au cours d'une semaine habituelle, combien de jours est-il physiquement actif pendant au moins 60 minutes? »). Les choix de réponse étaient les suivants :
(1) jamais (aucun jour), (2) 1 jour, (3) 2 ou 3 jours et (4) 4 jours ou plus. L'enfant était considéré comme actif physiquement si la réponse était (4), sinon il était considéré comme inactif $^{22}$. En ce qui concerne les adolescents de 12 à 17 ans, le module sur l'activité physique destiné aux adultes a été utilisé, et les niveaux d'activité physique étaient répartis en « actif », « modérément actif » et « inactif ${ }^{22,23}$. L'usage du tabac chez les adolescents a été catégorisé en « fumeurs actifs » (quotidiens ou occasionnels) et « non-fumeurs » (n'ayant jamais fumé).

L'indice de masse corporelle (IMC) a été calculé à partir de la mesure de la taille en position debout et du poids, et le statut pondéral a été établi en suivant les valeurs de référence pour la croissance des enfants d'âge scolaire et des adolescents de l'Organisation mondiale de la santé ${ }^{24}$. La circonférence de la taille a été mesurée au point médian entre le haut de la crête iliaque et la dernière côte flottante 22 .

La capacité aérobique et la capacité cardiorespiratoire ont été déterminées au moyen du Physitest aérobie canadien modifié (PACm), en consignant la fréquence cardiaque maximale prévue selon l'âge du participant et en calculant sa puissance aérobie maximale prévue $\left(\mathrm{VO}_{2}\right.$ $\max )^{25}$. La définition d'une « faible capacité aérobique » a été appliquée lorsque la valeur d'une variable calculée indiquant la condition aérobique se situait dans un intervalle généralement associé à certains risques pour la santé des enfants. Les détails concernant le calcul du score de capacité aérobique (SCA) et les normes relatives à la capacité aérobique sont énoncés dans des articles méthodologiques et dans le Guide de l'utilisateur des données de l'ECMS ${ }^{22,26,27}$. La PA systolique et la PA diastolique au repos ont été mesurées en suivant le nouveau protocole de mesure normalisée de la PA au cours d'enquêtes, qui est décrit dans une publication précédente ${ }^{28}$.

Toutes les analyses de laboratoire ont été réalisées par Santé Canada. Les détails relatifs aux procédures de laboratoire normalisées sont disponibles en ligne ${ }^{22}$. Les valeurs des variables déterminées en laboratoire qui étaient inférieures au seuil de détection ont été remplacées par le seuil de détection divisé par deux. Les variables dépendantes relatives aux marqueurs du risque de MCV dans l'échantillon complet étaient les taux de cholestérol total (en $\mathrm{mmol} / \mathrm{L}$ ), de cholestérol HDL (en mmol/L) et de protéine $\mathrm{C}$ réactive (CRP; en $\mathrm{mg} / \mathrm{L}$ ). Dans l'échantillon des participants à jeun, ces variables étaient les taux de cholestérol LDL (en $\mathrm{mmol} / \mathrm{L}$ ), de triglycérides (en $\mathrm{mmol} / \mathrm{L}$ ), de glucose (en mmol/L) et d'insuline (en $\mathrm{pmol} / \mathrm{L}$ ). La résistance à l'insuline a été estimée au moyen du modèle d'évaluation homéostasique de la résistance à l'insuline (HOMA-IR), une mesure indirecte de la résistance à l'insuline chez les enfants non diabétiques, qui est calculée en divisant par 22,5 le produit du taux de glucose (mmol/mL) et du taux d'insuline (pmol/ $\mathrm{mL})$ multiplié par $0,1394^{29}$. Afin de maintenir la taille de l'échantillon, nous avons exclu les répondants ayant des valeurs manquantes pour une variable dépendante donnée uniquement des analyses faisant appel à cette variable.

\section{Analyses statistiques}

Nous avons décrit les caractéristiques relatives au SSE et au risque de MCV des répondants pour les enfants et les adolescents et nous avons comparé garçons et filles au moyen de tests $t$. Nous avons d'abord examiné la corrélation polychorique entre la suffisance du revenu du ménage et le niveau de scolarité des parents. Comme ces variables n'étaient que modérément corrélées (coefficient de corrélation de 0,45 ), les effets indépendants de la suffisance du revenu et du niveau de scolarité des parents sur le risque de MCV ont été examinés d'abord par régression logistique multidimensionnelle pour l'excès de poids (y compris l'obésité) et la faible capacité aérobique, puis par régression linéaire multidimensionnelle pour l'IMC, la circonférence de la taille, le SCA, la PA, les lipides sanguins (totaux, cholestérol LDL et HDL, triglycérides), l'HOMA-IR, les taux de glucose et d'insuline à jeun et enfin le taux de CRP, après ajustement en fonction de l'âge et de l'origine ethnique et après stratification par sexe. En ce qui concerne les associations entre le SSE et la PA, les modèles ont également été ajustés en fonction de la 
fréquence cardiaque et de la taille. Nous n'avons pas tenu compte du poids à la naissance et de l'activité physique chez les enfants, ni de l'usage du tabac et de l'activité physique chez les adolescents, parce que ces variables pourraient avoir un effet médiateur et faire en sorte que les répercussions du SSE sur le risque de $\mathrm{MCV}$ soient sous-estimées dans ces tranches d'âge.

Étant donné la complexité du plan d'échantillonnage de l'ECMS et le nombre limité d'unités primaires d'échantillonnage aux cycles 1 et 2 , une pondération bootstrap a été appliquée pour l'estimation de la variance relative aux proportions, aux moyennes et aux paramètres des modèles de régression, avec 24 degrés de liberté déterminés pour les données combinées des cycles 1 et 2 . Nous avons effectué des analyses de régression logistique et de régression linéaire multidimensionnelles afin d'étudier les tendances relatives au risque de $\mathrm{MCV}$ dans les différentes strates de la suffisance du revenu du ménage et du niveau de scolarité des parents, après ajustement en fonction de l'âge et de l'origine ethnique. Des statistiques chi carré ajustées selon la méthode de Satterthwaite ont servi à déterminer la signification statistique $(p<0,05)$ d'une tendance linéaire.

Toutes les analyses ont été réalisées au moyen de la version 9.3 du logiciel SAS (SAS Institute Inc., Cary, Caroline du Nord, États-Unis) et de la version 10.0.1 du logiciel SUDANN (RTI International, Research Triangle Park, Caroline du Nord, États-Unis).

\section{Résultats}

Le tableau 1 montre les indicateurs du SSE et les facteurs de risque de MCV selon le sexe chez les enfants de 6 à 11 ans. À la naissance, le poids des garçons était significativement plus élevé que celui des filles. La prévalence de l'obésité chez les garçons $(18,5 \%$; intervalle de confiance [IC] à $95 \%$ : 15,6 à 21,5) était plus du double de celle observée chez les filles $(7,7 \%$; IC à $95 \%: 5,2$ à 10,3; $p<0,001)$. De même, la circonférence moyenne de la taille des garçons était supérieure à celle des filles $(62,5 \mathrm{~cm}$; IC à $95 \%: 61,7$ à
63,0 contre $60,4 \mathrm{~cm}$; IC à $95 \%: 59,7$ à $61,5 ; p=0,0004)$.

Les garçons étaient en meilleure condition physique que les filles, ainsi qu'en témoignent leur SCA moyen supérieur (541; IC à $95 \%$ : 534 à 546 contre 501; IC à $95 \%$ : 498 à 505; $p<0,001$ ) et la prévalence plus faible d'une faible capacité aérobique dans ce groupe $(23,2$; IC à $95 \%: 18,5$ à 28,7 contre 30,6; IC à $95 \%: 26,3$ à 35,3; $p=$ $0,04)$. Aucune différence entre les sexes n'a été observée en ce qui concerne la plupart des marqueurs physiologiques du risque de $\mathrm{MCV}$, exception faite du taux moyen d'insuline à jeun, qui était plus élevé chez les filles que chez les garçons $(54,6 \mathrm{pmol} / \mathrm{L}$; IC à $95 \%: 50,0$ à 59,2 contre $43,4 \mathrm{pmol} / \mathrm{L}$; IC à $95 \%: 37,6$ à 49,$2 ; p=0,03)$. Nous n'avons relevé aucune différence marquée entre les sexes pour ce qui est des indicateurs du SSE.

Le tableau 2 présente les indicateurs du SSE et les facteurs de risque de MCV selon le sexe chez les adolescents de 12 à 17 ans. L'écart entre les sexes concernant la capacité physique aérobique était au moins égal sinon supérieur chez les adolescents : les adolescentes affichaient des SCA encore plus faibles (460; IC à $95 \%$ : 454 à 466 contre 522; IC à $95 \%$ : 514 à 529 pour les garçons adolescents; $p<0,001$ ) et un taux supérieur de faible capacité aérobique par rapport aux garçons du même âge $(18,0$; IC à $95 \%$ : 14,7 à 21,9 pour les filles contre 8,60 ; IC à $95 \%: 5,97$ à 12,$3 ; p=0,002)$. En outre, nous avons observé des différences marquées en fonction du sexe pour plusieurs marqueurs du risque de MCV chez les adolescents : une PA systolique, un taux de glycémie à jeun et un taux de HDL défavorables chez les garçons, et des taux de cholestérol total et de CRP défavorables chez les filles. Là encore, aucune différence marquée entre les sexes n'a été observée en ce qui concerne les indicateurs du SSE.

Le tableau 3 présente les gradients du risque de MCV chez les enfants selon la suffisance du revenu et le niveau de scolarité des parents. Pour que la puissance statistique soit suffisante, nous avons combiné les groupes de l'excès de poids et de l'obésité en tant que variable dépendante dans les analyses de régression. Nous avons constaté l'existence d'un gradient lié au revenu significatif relativement à l'IMC ( $p$ pour la tendance : 0,006) et à l'excès de poids ( $p$ pour la tendance : 0,01 ) chez les jeunes filles, tandis que les jeunes garçons d'origine ethnique autre que blanche présentaient un risque accru d'excès de poids par rapport aux garçons d'origine ethnique blanche (rapport de cotes $[\mathrm{RC}]: 1,55$; IC à $95 \%: 1,03$ à $2,32)$. De plus, nous avons constaté l'existence de gradients liés au revenu et au niveau de scolarité en ce qui touche la capacité aérobique (SCA moyen et taux de faible capacité aérobique) chez les jeunes filles ( $p$ pour la tendance : 0,006 et 0,003, respectivement). La capacité aérobique des jeunes garçons présentait un gradient lié au revenu qui suivait une tendance similaire, mais la signification statistique n'a pas été atteinte ( $p$ pour la tendance : 0,11). Des gradients négatifs ont été observés en ce qui concerne la PA systolique et la PA diastolique élevées chez les jeunes enfants en fonction du niveau de scolarité, et indépendamment du sexe. Les enfants d'origine ethnique autre que blanche avaient un taux de cholestérol HDL moyen supérieur à celui des enfants d'origine ethnique blanche $(\beta=0,11$; IC à $95 \%: 0,04$ à $0,18)$. De plus, nous avons constaté un gradient positif relativement au taux de cholestérol HDL des jeunes filles en fonction du niveau de scolarité des parents ( $p$ pour la tendance : 0,047).

Le tableau 4 présente les gradients du risque de MCV chez les adolescents selon la suffisance du revenu et le niveau de scolarité des parents. Alors que nous n'avions pas décelé de gradient socioéconomique du risque d'excès de poids chez les jeunes garçons, nous avons observé un gradient positif lié au revenu chez les garçons adolescents, les garçons vivant dans les ménages à revenu supérieur affichant le plus haut risque. Nous avons constaté l'existence d'un gradient similaire pour ce qui est de la suffisance du revenu lorsque l'IMC était analysé en tant que variable continue. Le risque d'excès de poids suivait un gradient lié au niveau de scolarité chez les adolescentes, l'excès de poids touchant davantage les filles dont les parents avaient un faible niveau de scolarité. Les gradients socioéconomiques de la capacité aérobique persistaient chez les filles à l'adolescence ( $p$ pour la tendance : 0,05 ).

Pour ce qui est des autres marqueurs physiologiques du risque de $\mathrm{MCV}$, nous 
TABLEAU 1

Statut socioéconomique et risque cardiovasculaire chez les enfants canadiens (6 à 11 ans), selon le sexe

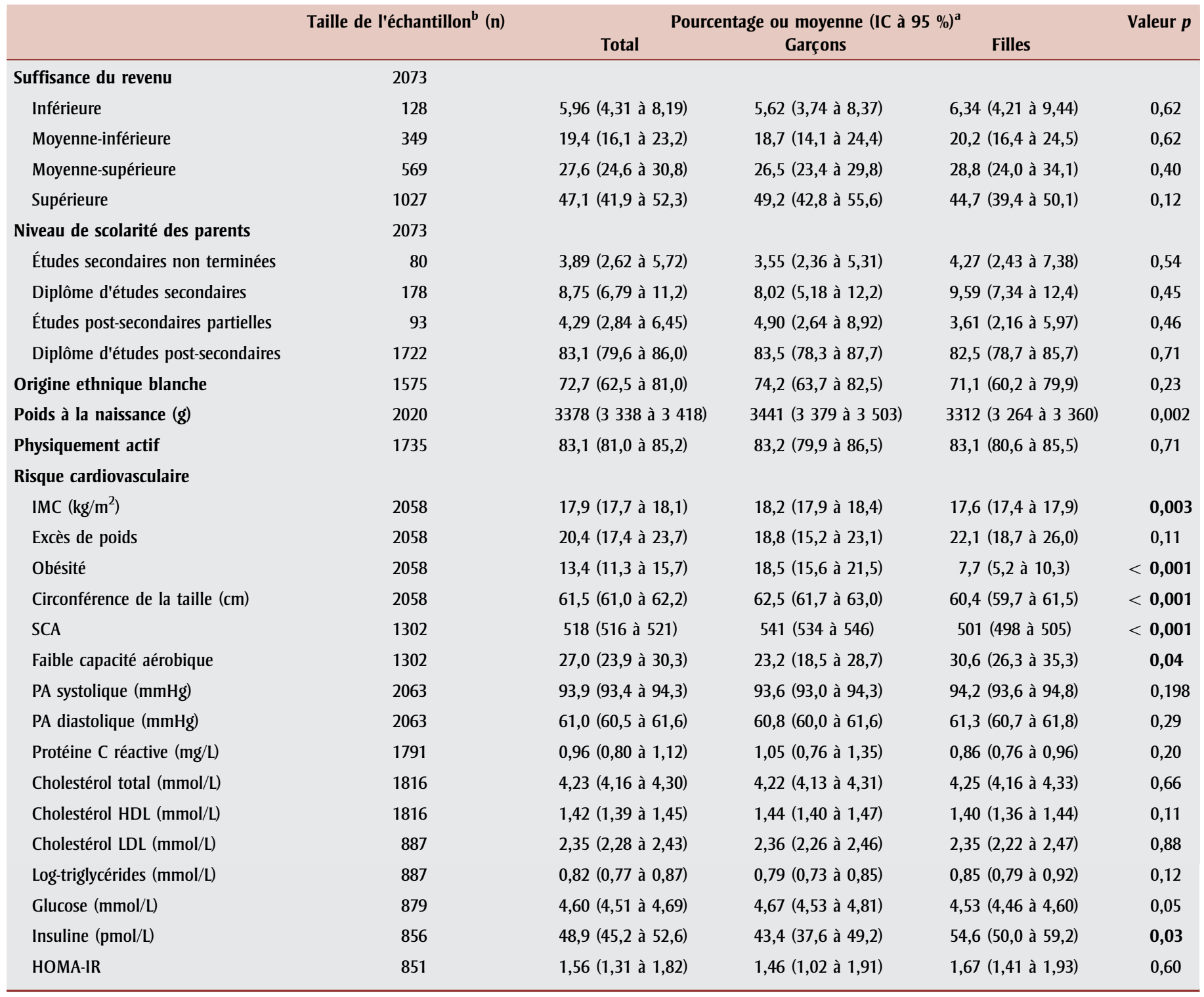

Source : Enquête canadienne sur les mesures de la santé, 2007-2011.

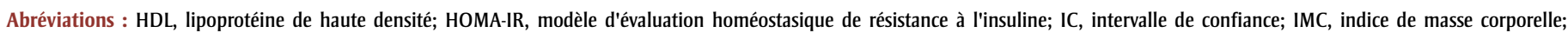
LDL, lipoprotéine de basse densité; PA, pression sanguine; SCA, score de capacité aérobique.

a Pourcentage ou moyenne pondérée selon la population, intervalle de confiance à $95 \%$.

b Selon la variable, 76 à 333 non-répondants de l'échantillon complet et 26 à 62 non-répondants de l'échantillon des sujets à jeun ont été exclus de l'analyse.

avons observé des gradients liés à la suffisance du revenu pour la PA systolique et les taux de cholestérol total et LDL chez les garçons adolescents, ceux vivant dans les ménages à revenu supérieur affichant le plus haut risque, cette tendance allant dans le même sens que le gradient lié au revenu observé pour l'excès de poids. De plus, le taux de cholestérol LDL des garçons adolescents suivait un gradient lié au niveau de scolarité, tandis que le taux de cholestérol HDL des adolescentes suivait un gradient lié au revenu, les adolescentes à faible SSE présentant le risque le plus élevé. De façon similaire à ce que nous avons observé chez les jeunes enfants, les adolescents d'origine ethnique autre que blanche avaient un taux moyen de cholestérol HDL supérieur à celui des adolescents d'origine ethnique blanche $(\beta=0,08$, IC à $95 \%: 0,01$ à 0,16$)$. Bien que nous ayons constaté des tendances significatives pour certains marqueurs du risque de MCV estimés au moyen des échantillons de sujets à jeun, ces résultats doivent être interprétés avec prudence, en raison de la petite taille des échantillons dans les groupes à faible SSE.

\section{Analyse}

Notre étude visait à déterminer l'existence de gradients socioéconomiques des marqueurs physiologiques du risque de MCV dans un échantillon représentatif à l'échelle nationale d'enfants et d'adolescents canadiens. Nous avons constaté l'existence d'importants gradients selon le sexe et le SSE en ce qui 
TABLEAU 2

Statut socioéconomique et risque cardiovasculaire chez les adolescents canadiens (12 à 17 ans), selon le sexe

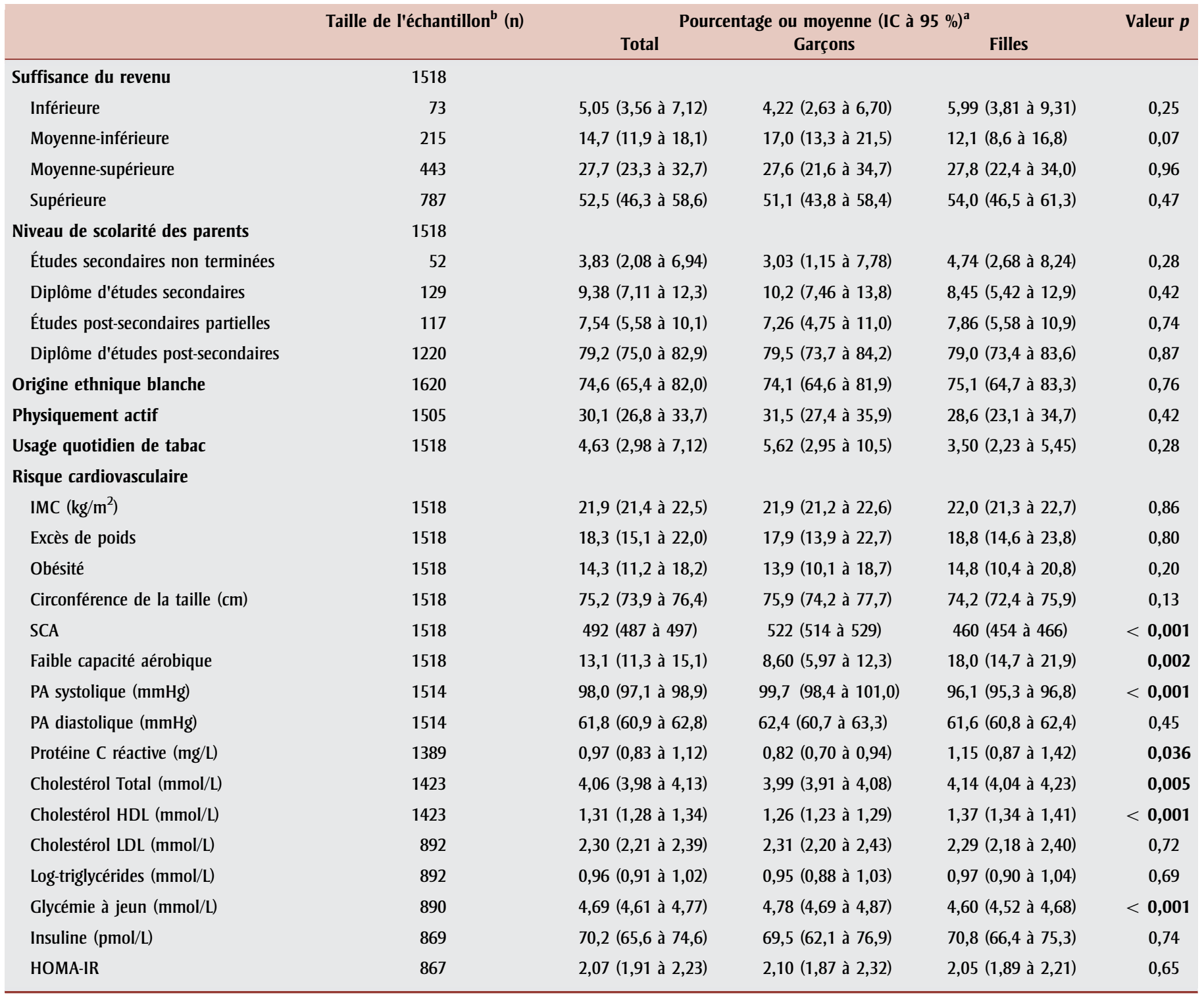

Source : Enquête canadienne sur les mesures de la santé, 2007-2011.

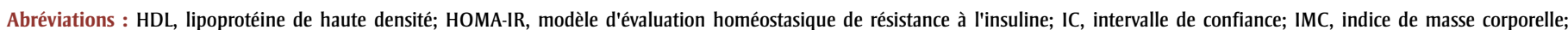
LDL, lipoprotéine de basse densité; PA, pression sanguine; SCA, score de capacité aérobique.

a Pourcentage ou moyenne pondérée selon la population, intervalle de confiance à $95 \%$.

b Selon la variable, 132 à 227 non-répondants de l'échantillon complet et 22 à 45 non-répondants de l'échantillon des sujets à jeun ont été exclus de l'analyse.

concerne l'adiposité et la capacité aérobique, gradients faisant leur apparition tôt dans l'enfance. La probabilité d'obésité était deux fois plus grande chez les jeunes garçons que chez les jeunes filles, et la diminution du risque d'excès de poids dans des conditions socioéconomiques favorables n'était observable que chez les filles. Plus important encore, nous avons constaté l'existence de gradients socioéconomiques en ce qui concerne la capacité aérobique tout au long de l'enfance, en particulier chez les filles. Les gradients liés au niveau de scolarité pour ce qui est de la PA font leur apparition tôt dans l'enfance. Alors que les garçons adolescents affichaient un risque plus grand de certains marqueurs physiologiques du risque de MCV dans les familles aisées, nous avons observé des gradients socioéconomiques en ce qui concerne un bas taux de cholestérol HDL chez les adolescentes et un taux élevé de cholestérol LDL chez les garçons adolescents, ceux à faible SSE présentant le risque le plus élevé.

Bon nombre d'études ayant eu pour but d'évaluer la relation entre le SSE et l'excès de poids chez les enfants et les adolescents des pays développés ont mis en lumière un gradient inverse entre le SSE et l'excès de poids ${ }^{30}$. L'association inverse que nous 


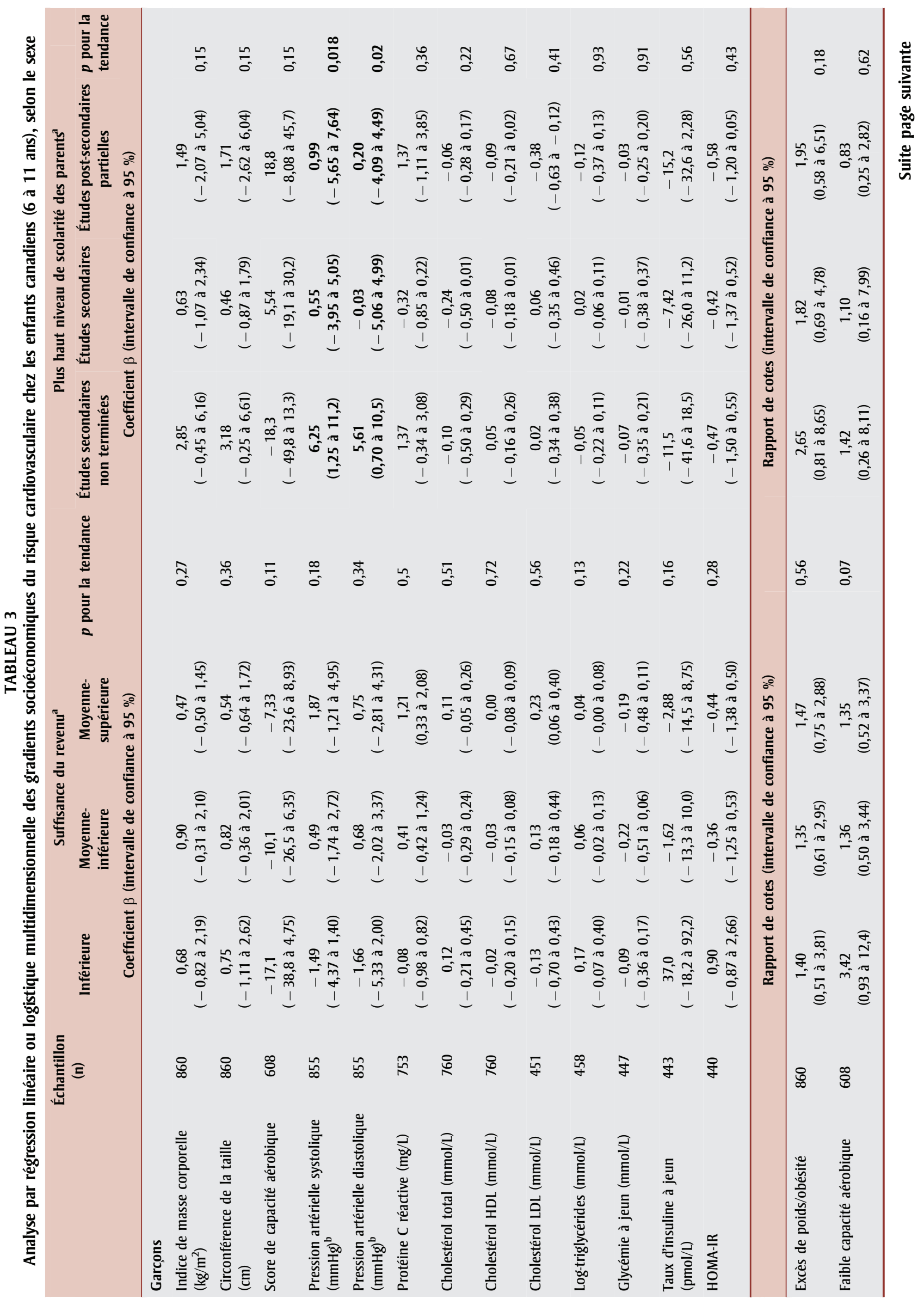




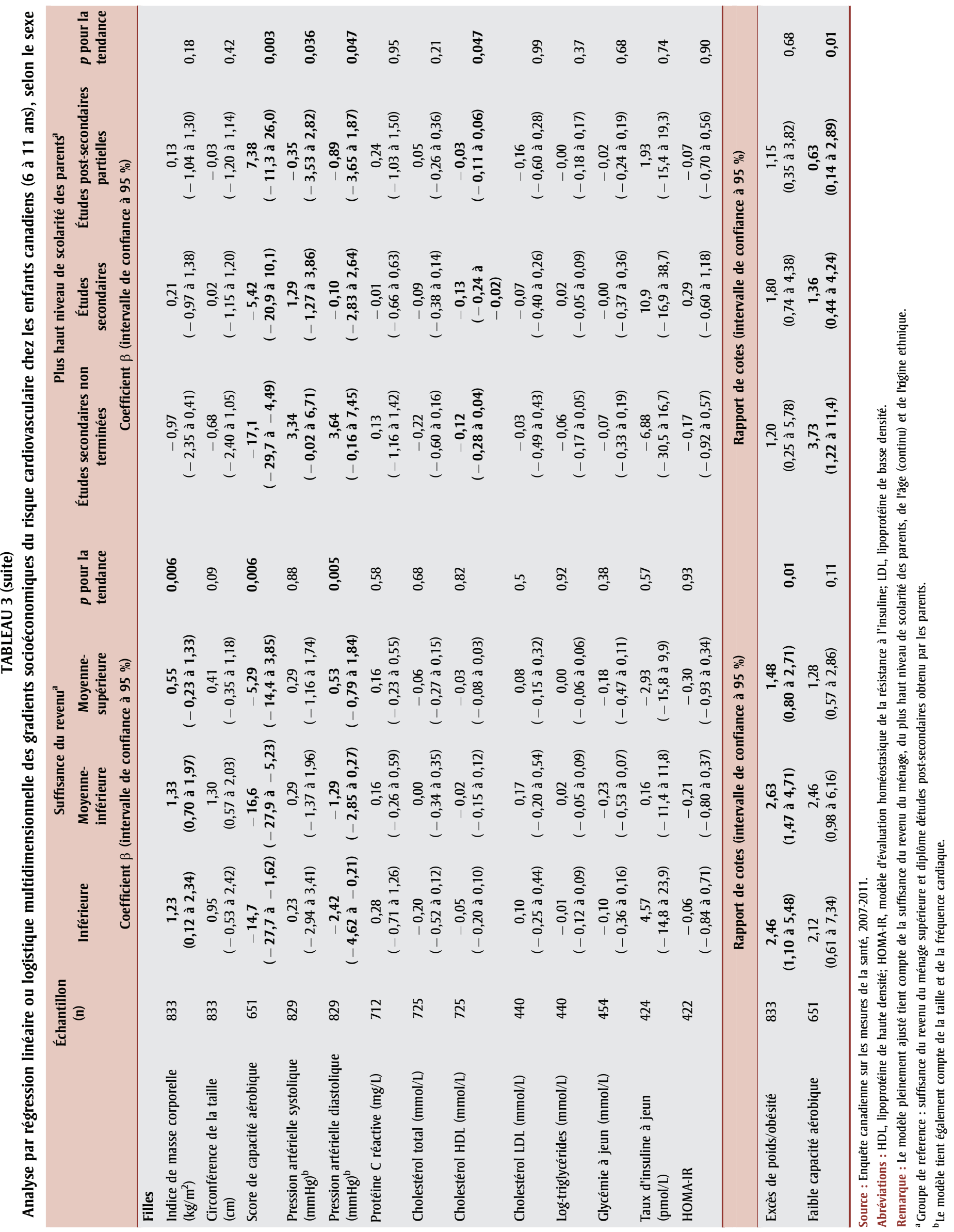




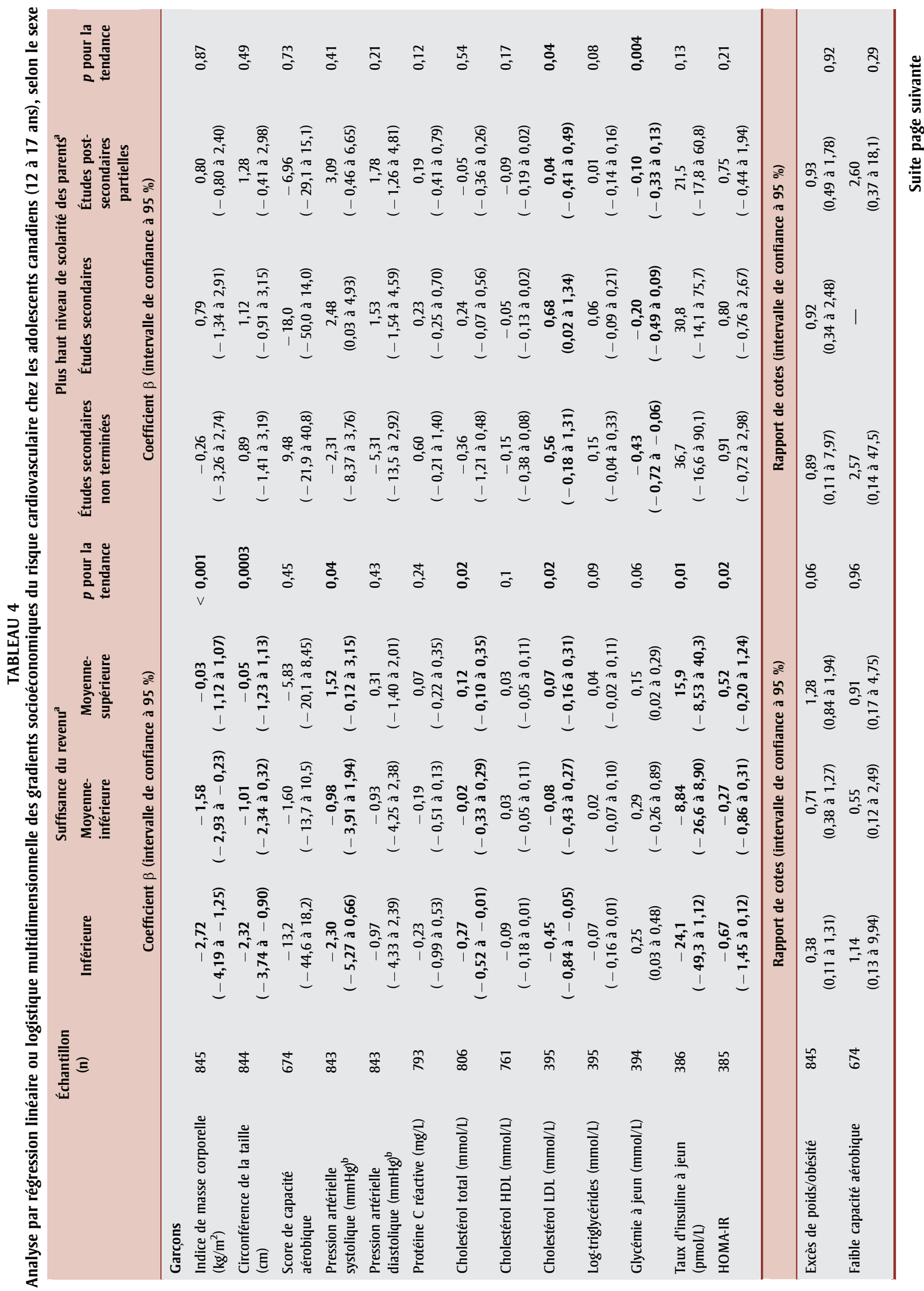




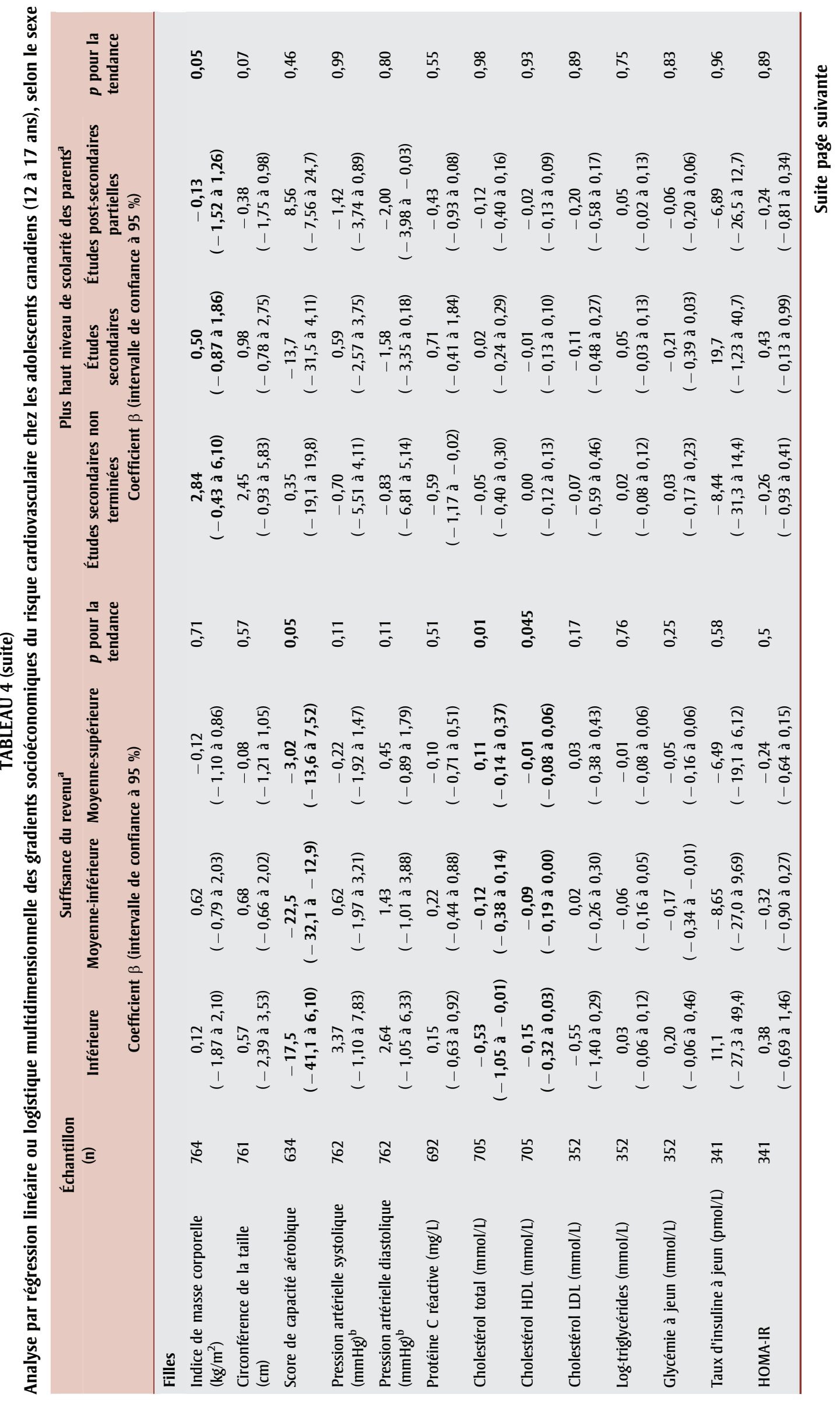




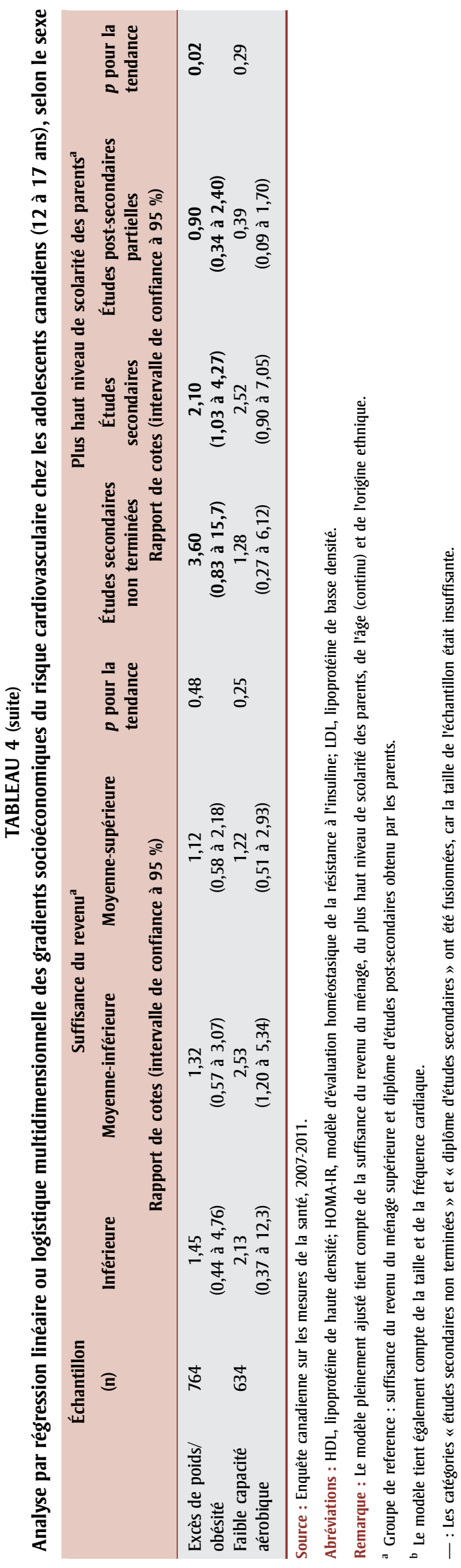

avons observée entre la suffisance du revenu et le risque d'excès de poids rejoint les résultats d'autres études canadiennes. Une étude menée chez des enfants de $6^{\mathrm{e}}$ à $10^{\mathrm{e}}$ année a révélé que les mesures du SSE tant à l'échelle individuelle que régionale étaient associées à l'obésité ${ }^{7}$, et une étude chez des élèves de $5^{\mathrm{e}}$ année dans une école de la Nouvelle-Écosse a fait état de résultats similaires ${ }^{16}$. À l'inverse, Shields et ses collaborateurs ${ }^{31}$ n'ont pas observé d'association de ce type avec les données d'enquête nationale qu'ils ont utilisées.

Dans ces études concernant le SSE et l'excès de poids chez les enfants, les auteurs n'ont procédé à aucune analyse selon le sexe. Nos résultats - un profil spécifique selon le sexe, et des gradients liés à la suffisance du revenu plus fortement associés à l'excès de poids chez les jeunes filles que chez les garçons sont comparables aux résultats d'études canadiennes récentes chez les adultes dans lesquelles les gradients socioéconomiques du risque d'excès de poids étaient plus marqués chez les femmes que chez les hommes $^{32,33}$. Il est généralement admis que les enfants de familles à faible revenu ont un accès limité à des ressources matérielles et peuvent moins se permettre de pratiquer des activités de loisir telles que les sports organisés, et il est aussi admis que la participation à des sports organisés favorise la perte de poids. Dans notre étude, nous n'avons pas pu déterminer si les filles sont plus vulnérables à la vie dans un environnement socioéconomique défavorisé, si cet impact débute tôt dans l'enfance ou si, en fait, les filles des familles aisées sont plus influencées et subissent davantage de pressions sociales pour demeurer minces dès leur jeune âge $\mathrm{A}^{34}$. De plus, l'absence de gradient socioéconomique relativement à l'excès de poids chez les jeunes garçons ne devrait pas amoindrir l'ampleur de la forte prévalence de l'obésité dans cette population. L'absence de gradient socioéconomique concernant l'excès de poids chez les jeunes garçons pourrait être attribuable à des attitudes culturelles ou sociales tolérantes à l'égard de l'excès de poids chez les garçons de toutes les catégories de revenu dans certains groupes ethniques ou raciaux. La constatation que nous avons faite que les garçons d'origine ethnique autre que blanche présentaient un risque plus grand 
d'excès de poids indépendamment du SSE vient étayer cette hypothèse. Nos résultats confirment le bien-fondé des programmes fédéraux, provinciaux ou territoriaux visant la réduction de l'obésité et le maintien d'un poids-santé chez les enfants $^{35}$. Ils montrent en outre que les interventions visant à prévenir l'obésité chez les jeunes et à réduire les gradients socioéconomiques de l'obésité devraient cibler non seulement les filles à faible SSE mais également les garçons de tous les groupes socioéconomiques.

Notre étude a aussi permis de mettre en lumière des différences marquées selon le sexe dans les gradients socioéconomiques relatifs à la capacité aérobique chez les enfants et les adolescents canadiens. Nous avons observé un gradient socioéconomique durable en ce qui concerne la capacité aérobique chez les filles canadiennes tout au long de l'enfance. La capacité aérobique (ou le SCA mesuré) utilisée dans notre étude est généralement considérée comme un effet physiologique d'une activité physique fréquente et comme un marqueur objectif de ce comportement ${ }^{20}$. Les mécanismes par lesquels un faible SSE pourrait avoir un effet sur la condition physique des enfants sont notamment des lacunes éducatives en matière de santé, la consommation d'aliments de piètre qualité et un accès limité à des installations récréatives.

Notre étude semble être la première à analyser la relation entre le SSE et la condition physique chez les jeunes Canadiens. Nos résultats sont similaires à ceux d'études menées chez des jeunes aux États-Unis $^{34}$ et en Suède ${ }^{36}$ ayant fait état de gradients socioéconomiques plus marqués chez les filles que chez les garçons en matière de condition physique. Certaines données laissent croire que les garçons sont plus nombreux à participer à des activités de loisir vigoureuses que les filles, et ce, quel que soit leur SSE, et que les filles sont plus nombreuses à pratiquer des activités physiques sous forme de sports organisés, auxquels la participation est davantage encouragée et appuyée dans les groupes à SSE élevé en $^{37,38}$. Cette hypothèse est corroborée par des études menées au Canada et ailleurs qui montrent que les filles des quartiers à faible SSE passent significativement plus de temps devant un écran que les filles vivant dans des quartiers à SSE élevé, relation qui n’a pas été observée chez les garçons ${ }^{39,40}$. Il importe de noter que les auteurs de certaines études ont avancé que les sports organisés constituaient l'un des meilleurs moyens d'encourager les adolescentes à pratiquer des activités vigoureuses ${ }^{41,42}$ et qu'une activité physique vigoureuse est le meilleur moyen d'avoir une bonne capacité cardiorespiratoire. Des recherches interventionnelles visant à trouver des moyens efficaces de promouvoir une bonne condition physique sont nécessaires pour réduire les gradients socioéconomiques chez les jeunes Canadiens, en particulier ceux des groupes à faible SSE.

Notre étude a mis en lumière des gradients socioéconomiques en ce qui concerne les biomarqueurs du risque de MCV chez les enfants canadiens, à savoir des gradients socioéconomiques en ce qui concerne la PA chez les jeunes enfants et les taux de cholestérol HDL et LDL chez les adolescents, ce qui laisse penser que des gradients socioéconomiques à vie du risque de MCV sont physiologiquement détectables dans l'enfance. La recherche intergénérationnelle donne à penser que le niveau de scolarité des parents peut influer indirectement sur la santé des enfants, à cause du revenu ou de son rôle sur le bienêtre psychologique de l'enfant, du fait d'un manque de compétences parentales ou d'un stress chronique, ce dernier pouvant être disproportionnellement élevé chez les enfants de familles défavorisées ${ }^{12}$. Notre observation d'un gradient lié au niveau de scolarité en ce qui concerne la PA rejoint les résultats d'autres études, qui ont conclu que le faible niveau de scolarité des parents et une vie dans un milieu familial difficile expliquaient en partie la variabilité de la PA chez les enfants ${ }^{43,44}$.

Les études ayant tenté d'élucider la relation entre le SSE et les lipides chez les enfants dans d'autres populations ont donné des résultats discordants ${ }^{45}$. Les données probantes actuelles laissent penser que l'origine ethnique, l'activité physique et le poids sont d'importants facteurs prédictifs du taux de cholestérol HDL et que la consommation d'aliments riches en gras saturés, l'activité physique et le poids sont des déterminants du taux de cholestérol LDL chez les enfants et les adolescents $^{46}$. Notre observation d'un profil HDL relativement favorable chez les personnes d'origine ethnique autre que blanche indépendamment du SSE semble indiquer que certains groupes ethniques sont génétiquement davantage protégés que d'autres contre les MCV. L'activité physique et le poids figurent parmi les déterminants du taux de cholestérol en bas âge, ce qui témoigne de l'utilité des messages de santé publique actuels visant à promouvoir l'activité physique et une alimentation saine et à prévenir l'excès de poids, en particulier chez les enfants des milieux défavorisés (et surtout les adolescents) afin de prévenir les disparités sur le plan du risque cardiovasculaire pouvant avoir leur source dans l'enfance.

\section{Points forts et points faibles}

Parmi les points forts de notre étude figure l'utilisation d'un échantillon représentatif à l'échelle nationale qui fournit une information fiable sur les mesures objectives du risque de $\mathrm{MCV}$, information qui nous a permis d'analyser les effets précoces du SSE sur les marqueurs physiologiques du risque de MCV. Cet échantillonnage à l'échelle de la population permet de généraliser les résultats à l'ensemble de la population canadienne.

Au nombre des points faibles de notre étude figurent la petite taille de l'échantillon, en particulier pour ce qui est des groupes à faible SSE et des participants qui ont subi des prélèvements de sang à jeun, ce qui a pu nous empêcher de détecter un gradient socioéconomique ou d'interpréter les tendances dans les sous-populations où les mesures étaient très variables, et a conduit à une incapacité à examiner les interactions possibles entre les indicateurs du SSE. Les biais associés aux renseignements autodéclarés sur le revenu familial et le niveau de scolarité des parents constituent une autre limite de notre étude. Comme il s'agissait d'une étude transversale, nous n'avons pas pu évaluer la modification avec le temps (hausse ou baisse) des gradients socioéconomiques du risque de $\mathrm{MCV}$ au cours de l'enfance. Certaines données laissent penser que les gradients socioéconomiques observés dans l'enfance an matière de santé se poursuivent 
jusqu'à l'âge adulte ${ }^{47}$, alors que d'autres données semblent indiquer que les gradients socioéconomiques établis dans l'enfance ne persistent pas à l'adolescence ${ }^{18}$. Ces conclusions divergentes témoignent de la nécessité de mener d'autres études sur le sujet.

\section{Conclusion}

Nous avons tenté de déterminer si les gradients socioéconomiques du risque de MCV clairement observés chez les adultes canadiens sont également présents chez les enfants et les adolescents. Nous avons analysé les associations indépendantes de la suffisance du revenu et du niveau de scolarité des parents avec les marqueurs physiologiques du risque de $\mathrm{MCV}$. Nous avons relevé des gradients marqués, selon le SSE et le sexe, en ce qui concerne l'adiposité et la capacité aérobique chez les enfants canadiens, en particulier chez les filles de 6 à 17 ans. En dépit de l'absence de gradient socioéconomique visible en ce qui concerne l'adiposité chez les garçons et, en fait, de l'existence d'un gradient socioéconomique inverse en ce qui a trait à l'excès de poids chez les garçons adolescents, les jeunes garçons affichaient une prévalence de l'obésité abdominale nettement supérieure à celle des filles. On ne devrait donc pas ignorer cette population dans les programmes visant à réduire le risque de MCV dans l'enfance.

Les gradients liés au niveau de scolarité observés chez les jeunes Canadiens en ce qui concerne certains marqueurs physiologiques sont à confirmer au moyen d'autres études. Les programmes de promotion de la santé devraient néanmoins sensibiliser les parents au risque de MCV précoce, en particulier les parents peu scolarisés.

Nos résultats confirment la pertinence des priorités actuelles que sont la réduction de l'obésité et des inégalités en matière de santé chez les enfants ${ }^{35}$. Il ressort également de notre étude que les interventions en santé publique visant à atténuer les gradients socioéconomiques du risque de MCV devraient être axées sur la réduction de l'obésité chez les jeunes et la promotion d'une bonne condition physique chez tous les enfants, et en particulier chez les filles et les groupes à faible SSE.

\section{Références}

1. Wielgosz A, Arango M, Bancej C et collab. (comité de rédaction). 2009 - Suivi des maladies du cœur et des accidents vasculaires cérébraux au Canada. Ottawa (Ont.) : Agence de la santé publique du Canada. 2009. PDF (10,6 Mo) téléchargeable à partir du lien : http://www.phac-aspc.gc.ca/publicat/ 2009/cvd-avc/pdf/cvd-avs-2009-fra.pdf

2. Choiniere R, Lafontaine P, Edwards AC. Distribution of cardiovascular disease risk factors by socioeconomic status among Canadian adults. CMAJ. 2000;162 (9 Suppl):S13-S24.

3. Millar WJ, Wigle DT. Socioeconomic disparities in risk factors for cardiovascular disease. CMAJ. 1986;134(2):127-132.

4. Batty GD, Leon DA. Socio-economic position and coronary heart disease risk factors in children and young people. Evidence from UK epidemiological studies. Eur J Public Health.2002;12(4):263-272.

5. Boreham C, Twisk J, van Mechalen W, Savage M, Strain J, Cran G. Relationships between the development of biological risk factors for coronary heart disease and lifestyle parameters during adolescence: The Northern Ireland Young Hearts Project. Public Health. 1999;113(1):7-12.

6. Elgar FJ, Pfortner TK, Moor I, De CB, Stevens $\mathrm{GW}$, Currie C. Socioeconomic inequalities in adolescent health 2002-2010: a time-series analysis of 34 countries participating in the Health Behaviour in School-aged Children study. Lancet. 2015;385(9982):2088-2095.

7. Janssen I, Boyce WF, Simpson K, Pickett W. Influence of individual- and area-level measures of socioeconomic status on obesity, unhealthy eating, and physical inactivity in Canadian adolescents. Am J Clin Nutr. 2006;83(1):139-145.

8. Simen-Kapeu A, Veugelers PJ. Les gradients socioéconomiques des habitudes de santé et du surpoids chez des enfants de milieux économiques distincts. Revue canadienne de santé publique. 2010;101(Suppl. 3)S34-S38.
9. Shonkoff JP, Boyce WT, McEwen BS. Neuroscience, molecular biology, and the childhood roots of health disparities: building a new framework for health promotion and disease prevention. JAMA. 2009;301(21):2252-2259. DOI: 10.1001/jama.2009.754.

10. Non AL, Rewak M, Kawachi I et collab. Childhood social disadvantage, cardiometabolic risk, and chronic disease in adulthood. Am J Epidemiol. 2014;180(3):263-271. DOI: $10.1093 / \mathrm{aje} / \mathrm{kwu} 127$.

11. Wannamethee SG, Whincup PH, Shaper G, Walker M. Influence of fathers' social class on cardiovascular disease in middle-aged men. Lancet. 1996;348(9037):1259-1263.

12. Chen E, Martin AD, Matthews KA. Trajectories of socioeconomic status across children's lifetime predict health. Pediatrics. 2007;120(2):e297-e303.

13. Bradley RH, Corwyn RF. Socioeconomic status and child development. Annu Rev Psychol. 2002;(53):371-399.

14. Richter M, Moor I, van Lenthe FJ. Explaining socioeconomic differences in adolescent self-rated health: the contribution of material, psychosocial and behavioural factors. J Epidemiol Community Health. 2012;66(8):691-697. DOI: 10.1136/jech.2010. 125500 .

15. Bambra CL, Hillier FC, Moore HJ, Summerbell $\mathrm{CD}$. Tackling inequalities in obesity: a protocol for a systematic review of the effectiveness of public health interventions at reducing socioeconomic inequalities in obesity amongst children. Syst Rev. 2012;1:16 DOI: 10.1186/ 2046-4053-1-16.

16. Veugelers PJ, Fitzgerald AL. Prevalence of and risk factors for childhood overweight and obesity. CMAJ. 2005;173(6):607-613.

17. Hajizadeh M, Campbell MK, Sarma S. Socioeconomic inequalities in adult obesity risk in Canada: trends and decomposition analyses. Eur J Health Econ. 2014;15(2): 203-221. DOI: 10.1007/s10198-013-0469-0.

18. Howe LD, Lawlor DA, Propper C. Trajectories of socioeconomic inequalities in health, behaviours and academic achievement across childhood and adolescence.J Epidemiol Community Health. 2013;67(4):358-364. 
19. McCrindle BW, Manlhiot C, Millar K et collab. Population trends toward increasing cardiovascular risk factors in Canadian adolescents. J Pediatr. 2010;157(5):837-843.

20. Van Lenthe FJ, Boreham CA, Twisk JW, Strain JJ, Savage JM, Smith GD. Socioeconomic position and coronary heart disease risk factors in youth. Findings from the Young Hearts Project in Northern Ireland. Eur J Public Health. 2001;11(1): 43-50.

21. Tremblay M, Wolfson M, Connor GS. Enquête canadienne sur les mesures de la santé : raison d'être, contexte et aperçu. Rapports sur la santé. 2007;18(Suppl.) 7-21.

22. Statistique Canada. Guide de l'utilisateur des données de l'Enquête canadienne sur les mesures de la santé (ECMS) : Cycle 2 [Internet]. Ottawa (Ont.) : Statistique Canada; 2012 [consultation le 26 février 2015]. Consultable en ligne à la page : http://www23.statcan.gc.ca/imdb-bmdi/ document/5071_D2_T1_V2-fra.htm

23. Craig CL, Marshall AL, Sjostrom M, et al. International physical activity questionnaire: 12 -country reliability and validity. Med Sci Sports Exerc. 2003;35(8): 1381-1395.

24. de Onis M, Onyango AW, Borghi E, Siyam A, Nishida C, Siekmann J. Development of a WHO growth reference for school-aged children and adolescents. Bull World Health Organ. 2007;85(9):660-667.

25. Société canadienne de physiologie de l'exercice (SCPE). Guide du conseiller en condition physique et habitudes de vie : programme «Santé et condition physique » de la Société canadienne de physiologie de l'exercice, $3^{\mathrm{e}}$ éd. Ottawa (Ont.) : Société canadienne de physiologie de l'exercice; 2004.
26. Shields M, Tremblay MS, Laviolette M, Craig CL, Janssen I, Connor Gorber S. Condition physique des adultes au Canada : résultats de l'Enquête canadienne sur les mesures de la santé, 2007-2009. Rapports sur la santé. 2010;21(1):23-38.

27. Tremblay MS, Shields M, Laviolette M, Craig CL, Janssen I, Connor Gorber S. Condition physique des enfants et des jeunes au Canada : résultats de l'Enquête canadienne sur les mesures de la santé de 2007-2009. Rapports sur la santé. 2010; 21(1):7-22.

28. Shi $\mathrm{Y}$, de Groh M, Morrison H. Increasing blood pressure and its associated factors in Canadian children and adolescents from the Canadian Health Measures Survey. BMC Public Health. 2012;12(1):388. DOI: 10.1186/ 1471-2458-12-388.

29. Lee JM, Okumura MJ, Davis MM, Herman WH, Gurney JG. Prevalence and determinants of insulin resistance among U.S. adolescents: a population-based study. Diabetes Care. 2006;29(11):2427-2432.

30. Sobal J, Stunkard AJ. Socioeconomic status and obesity: a review of the literature. Psychol Bull. 1989;105(2):260-275.

31. Shields M. L'embonpoint et l'obésité chez les enfants et les adolescents. Rapports sur la santé. 2006;17(3):27-43.

32. Hajizadeh M, Campbell MK, Sarma S. Socioeconomic inequalities in adult obesity risk in Canada: trends and decomposition analyses. Eur J Health Econ. 2014;15(2): 203-221. DOI: 10.1007/s10198-013-0469-0.

33. Matheson FI, Moineddin R, Glazier RH. The weight of place: a multilevel analysis of gender, neighborhood material deprivation, and body mass index among Canadian adults. Soc Sci Med. 2008;66(3): 675-690.
34. Bohr AD, Brown DD, Laurson KR, Smith PJ, Bass RW. Relationship between socioeconomic status and physical fitness in junior high school students. J Sch Health. 2013;83(8):542-7. DOI: 10.1111/josh.12063.

35. Agence de la santé publique du Canada. Pour un Canada plus sain : faire de la prévention une priorité : déclaration sur la prévention et la promotion de la part des ministres canadiens de la Santé, de la Promotion de la santé et du Mode de vie sain [Internet]. Ottawa (Ont.) : Agence de la santé publique du Canada [modification le 7 septembre 2010; consultation le 4 mars 2015]. Consultable en ligne à la page : http://www.phac-aspc.gc.ca/hp-ps/hl-mvs/ declaration/index-fra.php

36. Bergstrom E, Hernell O, Persson LA. Cardiovascular risk indicators cluster in girls from families of low socio-economic status. Acta Paediatr. 1996;85(9):1083-1090.

37. Jimenez-Pavon D, Kelly J, Reilly JJ. Associations between objectively measured habitual physical activity and adiposity in children and adolescents: systematic review. Int J Pediatr Obes. 2010;5(1):3-18. DOI: $10.3109 / 17477160903067601$.

38. Ness AR, Leary SD, Mattocks C et collab. Objectively measured physical activity and fat mass in a large cohort of children. PLoS Med. 2007;4(3):e97.

39. Carson V, Spence JC, Cutumisu N, Cargill L. Association between neighborhood socioeconomic status and screen time among pre-school children: a cross-sectional study. BMC Public Health. 2010;10:367. DOI: 10.1186/ 14712458-10-367.

40. Fairclough SJ, Boddy LM, Hackett AF, Stratton G. Associations between children's socioeconomic status, weight status, and sex, with screen-based sedentary behaviours and sport participation. Int J Pediatr Obes. 2009;4(4): 299-305. DOI: 10.3109/17477160902811215. 
41. Denton SJ, Trenell MI, Plotz T, Savory LA, Bailey DP, Kerr CJ. Cardiorespiratory fitness is associated with hard and light intensity physical activity but not time spent sedentary in 10-14 year old schoolchildren: the HAPPY study. PLoS One. 2013;8(4):e61073 DOI: 10.1371/journal.pone.0061073.

42. Phillips JA, Young DR. Past-year sports participation, current physical activity, and fitness in urban adolescent girls. J Phys Act Health. 2009;6(1):105-111.

43. Lehman BJ, Taylor SE, Kiefe CI, Seeman TE. Relationship of early life stress and psychological functioning to blood pressure in the CARDIA study. Health Psychol. 2009; 28(3):338-46. DOI: 10.1037/a0013785.

44. Pulkki L, Keltikangas-Jarvinen L, Ravaja N, Viikari J. Child-rearing attitudes and cardiovascular risk among children: moderating influence of parental socioeconomic status. Prev Med. 2003;36(1):55-63.

45. Donin AS, Nightingale CM, Owen CG et collab. Ethnic differences in blood lipids and dietary intake between UK children of black African, black Caribbean, South Asian, and white European origin: the Child Heart and Health Study in England (CHASE). Am J Clin Nutr. 2010;92(4):776-83. DOI: $10.3945 /$ ajcn.2010.29533.

46. Freedman DS, Strogatz DS, Williamson DF, Aubert RE. Education, race, and high-density lipoprotein cholesterol among US adults. Am J Public Health. 1992;82(7):999-1006.

47. Juhola J, Magnussen CG, Viikari JS et collab. Tracking of serum lipid levels, blood pressure, and body mass index from childhood to adulthood: the Cardiovascular Risk in Young Finns Study. J Pediatr. 2011;159(4): 584-590. 